\title{
Hepatic Sarcoidosis Presenting as Portal Hypertension and Liver Cirrhosis: Case Report and Review of the Literature
}

\author{
Christopher B. Tan ${ }^{a}$ Sadat Rashid ${ }^{c}$ Dhyan Rajan $^{a}$ \\ Wondwoosen Gebre $^{\text {b }}$ Paul Mustacchia ${ }^{c}$ \\ Departments of ${ }^{\mathrm{a}}$ Internal Medicine, ${ }^{\mathrm{b}} \mathrm{Pathology}$ and ${ }^{\mathrm{c}} \mathrm{G}$ astroenterology, \\ Nassau University Medical Center, East Meadow, N.Y., USA
}

\section{Key Words}

Hepatic sarcoidosis - Liver cirrhosis - Portal hypertension - Gastrointestinal sarcoidosis - Extrapulmonary sarcoidosis - Hepatic sarcoidosis and liver cirrhosis . Hepatic sarcoidosis and portal hypertension

\begin{abstract}
Systemic sarcoidosis is a disease of unknown etiology, with the liver being the third most commonly affected organ. Most cases of hepatic sarcoidosis are not clinically apparent, but a few can progress to liver cirrhosis, portal hypertension and ultimately liver failure. The diagnosis of hepatic sarcoidosis is difficult, considering that no single laboratory test or radiographic finding can definitively diagnose this systemic disease. Diagnosis of hepatic sarcoidosis relies heavily on histopathologic evaluation of two or more organs, a diagnostic modality that is invasive and may not be applicable to all patients. The treatment of hepatic sarcoidosis is challenging, with no large randomized controlled trials done to date. Physicians must be aware of the complications of hepatic sarcoidosis, and must include the same in the differential diagnosis of liver cirrhosis. We present a case of hepatic sarcoidosis complicated by portal hypertension and liver cirrhosis.
\end{abstract}

\section{Introduction}

Sarcoidosis is a systemic non-caseating granulomatous disease involving many organs [1]. The prevalence of sarcoidosis in the United States is reported to be 1-40 per 100,000 with a three-fold higher risk in African Americans when compared to Caucasians [2, 3]. Furthermore, there is increasing evidence of more severe disease in 
African Americans, with higher incidence of skin, eye, liver and bone marrow involvement.

The typical manifestation of sarcoidosis is the presence of non-caseating granulomas, affecting the lungs and lymph nodes in more than $90 \%$ of cases, but it can involve any organ system $[2,4,5]$. The liver is the third most commonly involved organ after the lymph nodes and the lungs $[3,4,6]$. Most cases of hepatic involvement from sarcoidosis are clinically silent, with only a few patients developing jaundice, chronic cholestasis, portal hypertension and/or Budd-Chiari syndrome [1,2]. Cirrhosis is a very rare complication of liver sarcoidosis found in less than $1 \%$ of cases $[4,7]$. We present a case of granulomatous liver disease secondary to sarcoidosis complicated with portal hypertension and liver cirrhosis.

\section{Case Report}

A 48-year-old African American male with a medical history significant for idiopathic pulmonary fibrosis, hypertension, coronary artery disease and gout was seen in the gastroenterology clinic for complaints of abdominal pain. The patient stated that for nearly 2 weeks he had been noticing intermittent, dull, right upper quadrant pain that was neither alleviated nor aggravated by any factors. He stated that he had had a decrease in appetite over the last 2 weeks and reported a 30 pound unintentional weight loss over the past 6 months. He denied the presence of any change in bowel habits, melena, hematemesis, vomiting, fever, chills or cough. He denied use of any tobacco, alcohol or recreational drugs. Family history was not significant, for the presence of any malignancy, gastrointestinal or pulmonary disorder. He was currently serving as a machine operator and did not report any hazardous occupational or environmental exposure.

Physical examination revealed a frail man in no acute distress. Abdominal examination yielded mild right upper quadrant tenderness without any guarding or rigidity. The liver edge was approximately 2-3 cm below the right costal margin, and an enlarged spleen was palpable. There were no stigmata of any chronic liver disease. Complete blood count performed was remarkable for leukopenia of $2,000 / \mathrm{mm}^{3}$ and thrombocytopenia of $109,000 / \mathrm{mm}^{3}$. Also noted was an alanine transaminase of $71 \mathrm{IU} / \mathrm{l}$ and an aspartate transaminase of $75 \mathrm{IU} / \mathrm{l}$, along with a markedly increased alkaline phosphatase of $898 \mathrm{IU} / \mathrm{l}$. Due to the abnormal liver-related tests, a laboratory evaluation of possible infectious, autoimmune and malignant liver processes was done, all yielding negative results (table 1). A computed tomography (CT) of the abdomen revealed hepatosplenomegaly with dilated portal and splenic vessels, suggestive of portal hypertension.

In consideration of the patient's laboratory and radiographic findings, a CT-guided biopsy of the liver was performed. Histopathologic evaluation of the biopsy specimen revealed non-caseating granulomatous inflammation with moderate periportal fibrosis, consistent with hepatic sarcoidosis (fig. 1). Special staining for fungus and acid-fast bacilli was negative. A subsequent CT of the thorax demonstrated bronchiectatic changes scattered in the lungs along with peripheral honeycombing, ground glass opacities and subpleural nodules with no significant mediastinal or hilar adenopathy. These findings were unchanged from a previous CT of the thorax performed a year earlier.

The patient was started on systemic steroids (prednisolone $0.5 \mathrm{mg} / \mathrm{kg} /$ day) which were continued for 6 weeks without improvement in clinical symptoms and liver tests. The patient was also referred for pulmonary evaluation to determine the presence of pulmonary sarcoidosis. Prior and recent CT thorax findings, laboratory values and pulmonary function testing were inconclusive for pulmonary sarcoidosis.

Upon follow-up 6 weeks later, his alkaline phosphatase and liver transaminases remained elevated, along with the presence of worsening leukopenia. The patient also complained of continuous abdominal pain, which had worsened from the initial visit. He now stated both right and left upper quadrant pain, with the latter appearing to be more severe. With no significant response to systemic steroids he was referred for splenectomy secondary to worsening pancytopenia, hypersplenism and abdominal discomfort. Splenectomy was also thought to be beneficial for this patient, considering the 
presence of portal hypertension in sarcoidosis. Gross examination of the spleen revealed it to be markedly enlarged, weighing approximately $990 \mathrm{~g}$ ( fig. 2a). Histopathologic examination of the splenic tissue showed diffuse confluent non-caseating granulomas and foreign body giant cells containing polarized foreign bodies with some patchy areas of calcification, highly consistent with sarcoidosis (fig. 2b). Special staining for fungus and acid-fast bacilli was negative.

After splenectomy, the patient continued to follow in hepatology, pulmonary and surgery clinics, and he displayed a marked improvement in his abdominal symptoms. A repeat CT scan of the abdomen 6 months later revealed mild diffuse heterogeneity of the liver parenchyma with diffuse nodularity of the liver contour, highly suggestive of cirrhosis ( $\underline{\text { fig. } 3}$ ). A small to moderate volume of complex ascites was also noted. With other causes of liver cirrhosis being excluded, a diagnosis of liver cirrhosis with portal hypertension secondary to hepatic sarcoidosis was made.

The patient was started on a low sodium diet and diuretics, which resulted in resolution of his ascites. Due to the presence of cirrhosis, he underwent a screening esophagogastroduodenoscopy which did not reveal any gastroesophageal varices. A follow-up liver ultrasonography (US) and alpha-fetoprotein level to evaluate for the presence of hepatocellular carcinoma were both negative. The patient is currently being evaluated for possible liver transplantation.

\section{Discussion}

Extrathoracic sarcoidosis can occur with and without lung involvement. It is rare to find isolated extrapulmonary disease, which accounts for less than $10 \%$ of cases [4]. Hepatic sarcoidosis mostly affects the younger population group between 20 and 40 years of age. Most of these lesions are usually asymptomatic, with only 5-30\% presenting with atypical clinical signs and symptoms including nausea, vomiting, jaundice, abdominal pain and hepatosplenomegaly [2, 4]. A small portion of hepatic sarcoidosis can be severe and rapidly progressive, with the occurrence of complications such as cirrhosis, portal hypertension, chronic cholestasis and Budd-Chiari syndrome $[2,6]$. The first report of portal hypertension associated with sarcoidosis was published in 1949 by Mino et al. [8], followed by Katskin in 1950 [9]. Since then, the incidence of sarcoidosis with portal hypertension and cirrhosis has gradually been increasingly reported [1]. Although half of patients with sarcoidosis may have portal hypertension without evidence of cirrhosis, early detection is crucial since the development of cirrhosis carries a poor prognosis [1].

Hepatic sarcoidosis can also manifest as chronic cholestasis, which can either be intra- or extrahepatic in nature. Chronic intrahepatic sarcoidosis may resemble primary biliary cirrhosis (PBC) and primary sclerosing cholangitis (PSC), and either of these two diseases can coexist with sarcoidosis $[1,6]$. Sarcoidosis is usually differentiated from PBC by normal IgM levels and the absence of elevated anti-mitochondrial antibody, which is positive in nearly $90 \%$ of patients with PBC. Sarcoidosis is differentiated from PSC by the absence of inflammatory bowel disease, lack of periductal fibrosis in histology, absence of anti-nuclear cytoplasmic antibody, restriction of the narrowing of the bile duct to a single area of the biliary system, and improvement with steroid treatment [1].

The pathophysiology of portal hypertension and cirrhosis in sarcoidosis is not completely understood and may involve multiple mechanisms. It has been suggested that small arterio-venous shunts may form in the region of the granulomas, resulting in an elevated portal blood flow which subsequently increases intrahepatic resistance $[1,3,10]$. Such resistance can also be increased by a large confluent of granulomas 
caused by healing fibrosis of the parenchyma in a haphazard distribution. The development of portal central bridging fibrosis may ultimately lead to cirrhotic remodeling and development of portal hypertension [1,5, 10]. Others suggest that the granulomas in the portal areas may produce pressure and restrict its normal flow, causing a perisinusoidal block $[1,11]$. Another theory suggests that cirrhosis and focal fibrosis are caused by ischemic changes brought about by primary granulomatous phlebitis of portal and hepatic veins, thereby increasing pre- and postsinusoidal resistance $[1,5]$. The pathogenesis of chronic intrahepatic cholestasis in sarcoidosis is due to destruction of bile ducts by portal and periportal granulomas that can ultimately lead to biliary cirrhosis [1].

The diagnosis of hepatic sarcoidosis is clinically challenging, considering its wide spectrum of disease presentation and the absence of any specific symptoms, signs, laboratory or radiographic findings. No precise guidelines are available for the assessment and diagnosis of hepatic sarcoidosis to date. It has been suggested that the diagnosis is made by clinical and radiologic findings suggestive of sarcoidosis, supported by histopathologic findings of non-caseating granulomas on biopsy obtained from either the liver, after the exclusion of other causes of hepatic granuloma formation such as lymphoma, solid tumors, Wegener's granulomatosis, PBC, farmer's lung disease, drug reactions and occupational/environmental exposures [12].

Routine laboratory evaluation in sarcoidosis is often non-specific. Laboratory studies such as peripheral lymphopenia with CD4 depletion, elevated ACE level, lysozyme, B-2 microglobulin, hypercalcemia and hypercalcuria can aid in the diagnosis $[4,6]$. Hepatic sarcoidosis usually demonstrates abnormal liver-related tests in $20-50 \%$ of cases, with alkaline phosphatase being most commonly affected [4, 5]. The degree of liver test abnormalities appears to be related to the degree of fibrosis and the degree of granulomatous inflammation [2].

Radiographic evaluation of abdominal sarcoidosis includes US, CT and magnetic resonance imaging (MRI) [13, 14]. Unlike thoracic sarcoidosis, which commonly presents with hallmark radiographic and CT findings of interstitial lung disease and mediastinal/hilar lymphadenopathy, no single imaging technique can accurately diagnose sarcoidosis in the abdomen [15]. US does not usually show hepatic or splenic focal sarcoidosis lesions, but it can demonstrate a non-specific increase in heterogeneity or echogenicity with organ enlargement, as well as the degree of fibrosis present in the granuloma $[13,16]$. In a recent study by Cremers et al. [2], US has a sensitivity of $46.2 \%$ in detecting hepatic sarcoidosis and has a limited value in confirming the diagnosis. CT scan of the abdomen may reveal diffuse hepatosplenomegaly, but focal hepatic and splenic lesions are only seen in 38 and 33\% of biopsy-proven cases, respectively [13]. Nodular hepatic sarcoidosis appears on CT as areas of multiple, low-attenuation lesions scattered throughout the liver, with no significant peripheral enhancement [16]. With MRI, the lesions are hyperintense on all sequences with lesser enhancement than the background liver parenchyma. These lesions are more conspicuous in gadolinium-enhanced MRI, which is considered superior to US and CT in the evaluation of abdominal sarcoidosis [13, 14].

The treatment of sarcoidosis with hepatic involvement remains controversial. The use of glucocorticoids may show some improvement of liver-related tests, but they do not alter the course and progression of the disease $[1,3,12]$. In our review of the 
literature, the most widely accepted approach in the treatment of hepatic sarcoidosis involves the use of glucocorticoids in patients with symptomatic sarcoidosis and evidence of cholestasis or elevated liver transaminases $[3,12]$. No treatment is necessary in asymptomatic patients [3,12]. Other alternate drugs reportedly being used in sarcoidosis include azathioprine, methotrexate, hydrochloroquine and infliximab [12]. No large randomized trials have been done to assess the efficacy of these drugs, which is most likely related to the rarity of the disease process. Patients with portal hypertension are also being treated with splenectomy [1]. Other Indications for splenectomy in sarcoidosis include symptomatic splenomegaly, severe hypersplenism, prophylaxis for splenic rupture and exclusion of neoplastic processes [12]. In advanced sarcoidosis of the liver, transplantation is the only option considered curative [12].

Although rare, the development of liver cirrhosis in patients with sarcoidosis should be considered in the differential diagnosis of cryptogenic cirrhosis. Clinicians should be aware of complications from hepatic sarcoidosis, as progression to cirrhosis harbors a poor prognosis.

Table 1. Laboratory evaluation of possible infectious, autoimmune and malignant liver processes

\begin{tabular}{ll}
\hline Laboratory test & Result (normal) \\
\hline Hepatitis B surface antigen & non-reactive \\
Hepatitis B core IgM antibody & non-reactive \\
Hepatitis B core antibody, total & non-reactive \\
Hepatitis B surface antibody, mIU/ml & $12(>10$, immunity) \\
Hepatitis A antibody IgM & non-reactive \\
Hepatitis A antibody, total & non-reactive \\
HCV antibody screening & non-reactive \\
Anti-mitochondrial antibodies & negative \\
Anti-smooth muscle antibodies & negative \\
Alpha-1-antitrypsin, mg/dl & $173(83-199)$ \\
Iron, $\mu$ g/dl & $56(45-160)$ \\
Transferrin saturation, \% & $18(20-55)$ \\
Ceruloplasmin, mg/dl & $47(18-36)$ \\
Alpha-fetoprotein, serum tumor marker, ng/ml & $2.1(<6.1)$ \\
\hline
\end{tabular}




\begin{tabular}{l|l|l|l}
$\begin{array}{r}\text { Case Reports in } \\
\text { Gastroenterology }\end{array}$ & $\begin{array}{l}\text { Case Rep Gastroenterol 2012;6:183-189 } \\
\text { DOI: 10.1159/000338355 }\end{array}$ & $\begin{array}{l}\text { Published online: } \\
\text { April 18, 2012 }\end{array}$ & $\begin{array}{l}\text { @ 2012 S. Karger AG, Basel } \\
\text { ISSN 1662-0631 } \\
\text { www.karger.com/crg }\end{array}$ \\
\hline
\end{tabular}

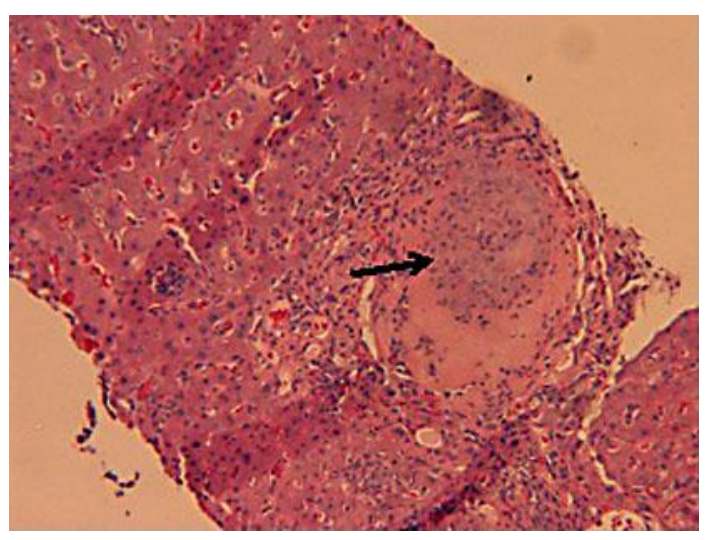

Fig. 1. Liver biopsy revealing the presence of a non-caseating granuloma (arrow) with epitheloid cells.
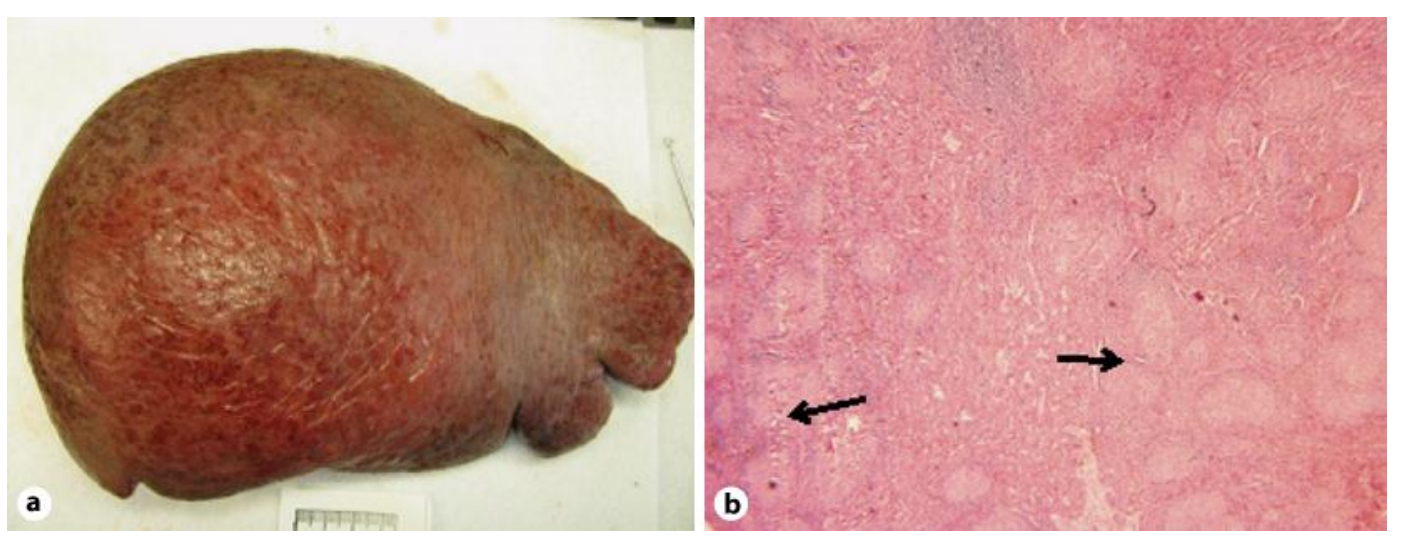

Fig. 2. a Enlarged spleen with multiple nodular pattern typical of sarcoidosis. b Microscopic specimen of the spleen revealing multiple non-caseating granulomas (arrows) composed of epitheloid cells and multi-nuclear giant cells. 


\begin{tabular}{r|l|l|l}
$\begin{array}{r}\text { Case Reports in } \\
\text { Gastroenterology }\end{array}$ & $\begin{array}{l}\text { Case Rep Gastroenterol 2012;6:183-189 } \\
\text { DOI: 10.1159/000338355 }\end{array}$ & $\begin{array}{l}\text { Published online: } \\
\text { April 18, 2012 }\end{array}$ & $\begin{array}{l}\text { @ 2012 S. Karger AG, Basel } \\
\text { ISSN 1662-0631 } \\
\text { www.karger.com/crg }\end{array}$ \\
\hline
\end{tabular}

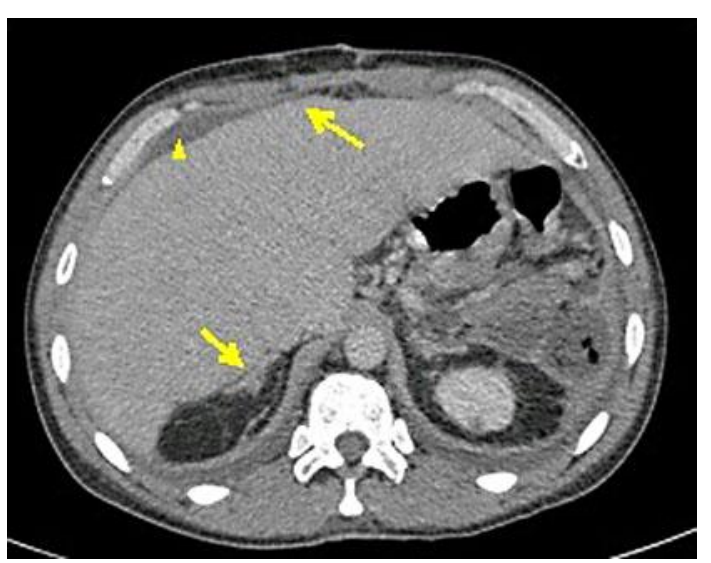

Fig. 3. CT scan of the abdomen. Note the diffuse heterogeneity of the liver parenchyma with diffuse nodularity of the liver contour (arrows), highly suggestive of liver cirrhosis. Mild ascites was also noted (arrowhead).

\section{References}

1 Blich M, Edoute Y: Clinical manifestations of sarcoid liver disease. J Gastroenterol Hepatol 2004;19: 732-737.

-2 Cremers J, et al: Liver-test abnormalities in sarcoidosis. Eur J Gastroenterol Hepatol 2012;24:17-24.

-3 Ebert EC, Kierson M, Hagspiel KD: Gastrointestinal and hepatic manifestations of sarcoidosis. Am J Gastroenterol 2008;103:3184-3192; quiz 3193.

4 Gavilan F, et al: Hepatic cirrhosis with sarcoid granulomas. Differential diagnosis and liver transplantation: a case report. Transplant Proc 2003;35:713-714.

5 Malhotra A, Naniwadekar A, Sood G: Hepatobiliary and pancreatic: cirrhosis secondary to hepatic sarcoidosis. J Gastroenterol Hepatol 2008;23:1942.

6 Pereira-Lima J, Schaffner F: Chronic cholestasis in hepatic sarcoidosis with clinical features resembling primary biliary cirrhosis. Report of two cases. Am J Med 1987;83:144-148.

7 Bass NM, et al: Chronic intrahepatic cholestasis due to sarcoidosis. Gut 1982;23:417-421.

-8 Mino RA, Murphy AI Jr, Livingstone RG: Sarcoidosis producing portal hypertension; treatment by splenectomy and splenorenal shunt. Ann Surg 1949;130:951-957.

9 Klatskin G, Yesner R: Hepatic manifestations of sarcoidosis and other granulomatous diseases; a study based on histological examination of tissue obtained by needle biopsy of the liver. Yale J Biol Med 1950;23:207-248.

10 Maddrey WC, et al: Sarcoidosis and chronic hepatic disease: a clinical and pathologic study of 20 patients. Medicine (Baltimore) 1970;49:375-395.

$\checkmark 11$ Valla D, et al: Hepatic sarcoidosis with portal hypertension. A report of seven cases with a review of the literature. Q J Med 1987;63:531-544.

12 Giovinale M, et al: Atypical sarcoidosis: case reports and review of the literature. Eur Rev Med Pharmacol Sci 2009;13(suppl 1):37-44.

13 Kessler A, et al: Hepatic and splenic sarcoidosis: ultrasound and MR imaging. Abdom Imaging 1993;18:159-163.

14 Dourakis SP, et al: A case of liver sarcoidosis mimicking cirrhosis. Clin Imaging 2007;31:47-49.

15 Scott GC, Berman JM, Higgins JL Jr: CT patterns of nodular hepatic and splenic sarcoidosis: a review of the literature. J Comput Assist Tomogr 1997;21:369-372.

16 Vagal AS, Shipley R, Meyer CA: Radiological manifestations of sarcoidosis. Clin Dermatol 2007;25: 312-325. 